\title{
Nuclear cusp conditions for components of the molecular energy density relevant for density-functional theory
}

\author{
N. H. March ${ }^{1,2}$ I. A. Howard, ${ }^{1}$ A. Holas, ${ }^{3}$ P. Senet, ${ }^{1}$ and V. E. Van Doren ${ }^{1}$ \\ ${ }^{1}$ Department of Physics, University of Antwerp (RUCA), Groenenborgerlaan 171, B-2020 Antwerp, Belgium \\ ${ }^{2}$ University of Oxford, Oxford, England \\ ${ }^{3}$ Institute of Physical Chemistry of the Polish Academy of Sciences, 44/52 Kasprzaka, 01-224 Warsaw, Poland
}

(Received 29 June 2000; published 14 December 2000)

\begin{abstract}
Nuclear cusp conditions are obtained for the full electron-electron interaction energy density as well as for the exchange and correlation energy densities of density-functional theory. Their form is the same as the form of the well known Kato cusp condition for the electron-number density. All these cusp conditions are valid for both the ground and excited states of a molecule or solid.
\end{abstract}

DOI: 10.1103/PhysRevA.63.012520

PACS number(s): 31.15.Ew, 31.10.+z, 71.15.Mb, 71.45.Gm

\section{INTRODUCTION}

A singularity at the nuclear position $\mathbf{R}_{A}$ of the electronnucleus interaction potential

$$
v_{A}(\mathbf{r})=-\frac{Z_{A}}{\left|\mathbf{r}-\mathbf{R}_{A}\right|}
$$

causes the $N$-electron wave function of a molecule to be a nonanalytic function of each electron-position variable $\mathbf{r}_{i}, i$ $=1,2, \ldots, N$, at $\mathbf{r}_{i}=\mathbf{R}_{A}$, but the function remains bounded (finite) everywhere. The character of this non-analyticity, known as the nuclear cusp condition, was determined for an atom by Kato [1]. His result was extended to a molecule by Bingel [2], and generalized by Pack and Brown [3] to the case where nuclear motion is included. For a molecule with fixed nuclear positions, as considered in the present investigation, Kato's cusp condition for the wave function leads (see Steiner [4]) to the following condition for the electronnumber density $n_{1}(\mathbf{r})$ :

$$
\left.\frac{d \overline{n_{1}}(r)}{d r}\right|_{r=0}=-2 Z_{A} n_{1}(\mathbf{0}),
$$

where $Z_{A}$ is the nuclear charge, while the center of the coordinate system is placed at the $A$ th nucleus position, $\mathbf{R}_{A}$ $=\mathbf{0}$. The angular average $\bar{F}(r)$ of a function $F(\mathbf{r})$ is defined by

$$
\bar{F}(r)=\int \frac{d^{2} \Omega}{4 \pi} F(r \mathbf{e}(\Omega)),
$$

where, in spherical coordinates, $\mathbf{r}=r \mathbf{e}(\Omega),|\mathbf{e}|=1$.

We are going to show that the nuclear cusp condition is satisfied not only by the electron-number density, Eq. (1.2), but also by other densities used for the calculation of the energy of a molecule. Such results may be relevant for density-functional theory (DFT) applications to both the ground and excited states (see Nagy [5]).

Consider a molecule as an $\mathrm{N}$-electron system described by the Hamiltonian

$$
\hat{H}=\hat{T}+\hat{V}+\hat{U},
$$

where $\hat{T}=\sum_{i=1}^{N} \hat{t}\left(\mathbf{r}_{i}\right), \hat{V}=\sum_{i=1}^{N} v\left(\mathbf{r}_{i}\right), \quad \hat{U}=\sum_{1 \leqslant i<j \leqslant N} u\left(\mathbf{r}_{i}\right.$, $\left.\mathbf{r}_{j}\right)$. Here $\mathbf{x}_{i} \equiv\left\{\mathbf{r}_{i}, s_{i}\right\}$ denotes the space-spin coordinate of the $i$ th electron, $\hat{t}(\mathbf{r})=-\frac{1}{2} \nabla^{2}(\mathbf{r})$ is the kinetic energy operator, $v(\mathbf{r})=\Sigma_{A} v_{A}(\mathbf{r})$ [Eq. (1.1)] is the external potential (due to clamped nuclei), and $u\left(\mathbf{r}_{i}, \mathbf{r}_{j}\right)=1 /\left|\mathbf{r}_{i}-\mathbf{r}_{j}\right|$ is the electronelectron interaction potential. Atomic units are used throughout. Let the $N$-electron, antisymmetric, normalized $(\langle\Psi \mid \Psi\rangle$ =1) wave function $\Psi\left(\mathbf{x}_{1}, \ldots, \mathbf{x}_{N}\right)$ be some eigenfunction of the Schrödinger equation

$$
\hat{H} \Psi=E \Psi .
$$

Discussing cusp conditions due to the particular $A$ th nucleus, we choose its position to be at $\mathbf{R}_{A}=\mathbf{0}$. Of all (equivalent) arguments of $\Psi$ we focus our attention on $\mathbf{r}_{1}=r_{1} \mathbf{e}\left(\Omega_{1}\right)$.

The presentation of the nuclear cusp problem given by Davidson [6] serves us now as a starting point of our investigation. Since $\Psi$ is finite everywhere, we have from Eq. (1.5)

$$
\lim _{r_{1} \rightarrow 0} r_{1} \hat{H} \Psi=0 .
$$

This means that $\Psi\left(\mathbf{r}_{1}, s_{1}, \mathbf{x}_{2}, \ldots, \mathbf{x}_{N}\right)$ is also the eigenfunction, with zero eigenvalue, of the operator

$$
\hat{b}\left(\mathbf{r}_{1}\right)=\lim _{r_{1} \rightarrow 0} r_{1} \hat{T}+\gamma=\lim _{r_{1} \rightarrow 0} r_{1} \hat{t}\left(\mathbf{r}_{1}\right)+\gamma,
$$

where

$$
\gamma=\lim _{r_{1} \rightarrow 0} r_{1}(\hat{V}+\hat{U})=\lim _{r_{1} \rightarrow 0} r_{1} v_{A}\left(\mathbf{r}_{1}\right)=-Z_{A} .
$$

For the wave function $\Psi\left(\mathbf{r}_{1}, \mathbf{X}\right)$ where $\mathbf{X} \equiv\left\{s_{1}, \mathbf{x}_{2}, \ldots, \mathbf{x}_{N}\right\}$, expanded in spherical harmonics

$$
\Psi\left(\mathbf{r}_{1}, \mathbf{X}\right)=\sum_{l=0}^{\infty} \sum_{m=-l}^{m=l} Y_{l m}\left(\Omega_{1}\right) r_{1}^{l} f_{l m}\left(r_{1}, \mathbf{X}\right)
$$


with $f_{l m}\left(r_{1}, \mathbf{X}\right)$ an analytic function of $r_{1}$, the eigenequation mentioned above,

$$
\hat{b}\left(\mathbf{r}_{1}\right) \Psi=0,
$$

leads to the following conditions $[3,6]$ on the partial waves amplitudes:

$$
\left.\frac{\partial f_{l m}\left(r_{1}, \mathbf{X}\right)}{\partial r_{1}}\right|_{r_{1}=0}=\frac{\gamma}{l+1} f_{l m}(0, \mathbf{X}) .
$$

Equations (1.8)-(1.11) are sufficient for obtaining all interesting cusp conditions. These for the system described by the Hamiltonian in Eq. (1.4) will be considered in Sec. II, and those for systems of adiabatic connection (a method that leads to the construction of DFT objects) in Sec. III. The implications of our results will be discussed in Sec. IV.

\section{RESULTS FOR THE FULLY INTERACTING SYSTEM}

As is well known (see, e.g., Davidson [6]) the total energy $E=\langle\Psi|\hat{H}| \Psi\rangle$ - the eigenenergy if $\Psi$ is the solution of the Schrödinger equation (1.5) - can be written in terms of the first and second order density matrix (1DM, 2DM) derived from $\Psi$, namely,

$$
\begin{aligned}
E= & T+V+E_{\mathrm{ee}}=\int d^{3} r_{1}\left\{\varepsilon_{\mathrm{kin}}\left(\mathbf{r}_{1} ;\left[\rho_{1}\right]\right)\right. \\
& \left.+v\left(\mathbf{r}_{1}\right) n_{1}\left(\mathbf{r}_{1}\right)+\varepsilon_{\mathrm{ee}}\left(\mathbf{r}_{1} ;\left[n_{2}\right]\right)\right\},
\end{aligned}
$$

where

$$
\varepsilon_{\text {kin }}\left(\mathbf{r}_{1} ;\left[\rho_{1}\right]\right)=\left.\hat{t}\left(\mathbf{r}_{1}\right) \rho_{1}\left(\mathbf{r}_{1} ; \mathbf{r}_{1}^{\prime}\right)\right|_{\mathbf{r}_{1}^{\prime}=\mathbf{r}_{1}}
$$

is the kinetic energy density, $v\left(\mathbf{r}_{1}\right) n_{1}\left(\mathbf{r}_{1}\right)$ is the electronnucleus interaction energy density, and

$$
\varepsilon_{\mathrm{ee}}\left(\mathbf{r}_{1} ;\left[n_{2}\right]\right)=\int d^{3} r_{2} u\left(\mathbf{r}_{1}, \mathbf{r}_{2}\right) n_{2}\left(\mathbf{r}_{1}, \mathbf{r}_{2}\right)
$$

is the electron-electron interaction energy density. The DMs $\rho_{1}, n_{2}$, and $n_{1}$ can be obtained from the 2DM

$$
\begin{aligned}
\rho_{2}\left(\mathbf{r}_{1}, \mathbf{r}_{2} ; \mathbf{r}_{1}^{\prime}, \mathbf{r}_{2}^{\prime}\right)= & \frac{N(N-1)}{2} \int d \mathbf{Q} \Psi\left(\mathbf{r}_{1}, \mathbf{r}_{2}, \mathbf{Q}\right) \\
& \times \Psi^{*}\left(\mathbf{r}_{1}^{\prime}, \mathbf{r}_{2}^{\prime}, \mathbf{Q}\right) .
\end{aligned}
$$

Here the notation $\mathbf{Q} \equiv\left\{s_{1}, s_{2}, \mathbf{x}_{3}, \ldots, \mathbf{x}_{N}\right\}$ is introduced to split the previous $\mathbf{X}$ as $\mathbf{X}=\left\{s_{1}, \mathbf{r}_{2}, s_{2}, \mathbf{x}_{3}, \ldots, \mathbf{x}_{N}\right\}=\left\{\mathbf{r}_{2}, \mathbf{Q}\right\}$, and $\int d \mathbf{Q}$ means summation over $s_{i}$ and integration over $d^{3} r_{i}$ for all variables included in $\mathbf{Q}$. Thus, the electron-pair density is the diagonal element of the 2DM,

$$
n_{2}\left(\mathbf{r}_{1}, \mathbf{r}_{2}\right)=\rho_{2}\left(\mathbf{r}_{1}, \mathbf{r}_{2} ; \mathbf{r}_{1}, \mathbf{r}_{2}\right),
$$

the $1 \mathrm{DM}$ is the reduced $2 \mathrm{DM}$,

$$
\rho_{1}\left(\mathbf{r}_{1} ; \mathbf{r}_{1}^{\prime}\right)=\frac{2}{N-1} \int d^{3} r_{2} \rho_{2}\left(\mathbf{r}_{1}, \mathbf{r}_{2} ; \mathbf{r}_{1}^{\prime}, \mathbf{r}_{2}\right)
$$

and the electron-number density is the diagonal element of the 1DM,

$$
n_{1}\left(\mathbf{r}_{1}\right)=\rho_{1}\left(\mathbf{r}_{1} ; \mathbf{r}_{1}\right)
$$

In order to establish the cusp condition for $n_{2}\left(\mathbf{r}_{1}, \mathbf{r}_{2}\right)$, let us calculate this DM using the expansion (1.9) for $\Psi$ and $\Psi^{*}$ in Eqs. (2.4), (2.5):

$$
\begin{aligned}
n_{2}\left(\mathbf{r}_{1}, \mathbf{r}_{2}\right)= & \frac{N(N-1)}{2} \int d \mathbf{Q} \sum_{l m l^{\prime} m^{\prime}} Y_{l m}\left(\Omega_{1}\right) Y_{l^{\prime} m^{\prime}}^{*}\left(\Omega_{1}\right) \\
& \times r_{1}^{l+l^{\prime}} f_{l m}\left(r_{1}, \mathbf{r}_{2}, \mathbf{Q}\right) f_{l^{\prime} m^{\prime}}^{*}\left(r_{1}, \mathbf{r}_{2}, \mathbf{Q}\right) .
\end{aligned}
$$

According to Eq. (1.3), its angular average is

$$
\begin{aligned}
\overline{n_{2}}\left(r_{1}, \mathbf{r}_{2}\right) & =\frac{1}{4 \pi} \int d^{2} \Omega_{1} n_{2}\left(r_{1} \mathbf{e}\left(\Omega_{1}\right), \mathbf{r}_{2}\right) \\
& =\frac{N(N-1)}{2} \int d \mathbf{Q} \frac{1}{4 \pi} \sum_{l m} r_{1}^{2 l}\left|f_{l m}\left(r_{1}, \mathbf{r}_{2}, \mathbf{Q}\right)\right|^{2} .
\end{aligned}
$$

The derivative of this function,

$$
\begin{aligned}
\frac{\partial \overline{n_{2}}\left(r_{1}, \mathbf{r}_{2}\right)}{\partial r_{1}}= & \frac{N(N-1)}{2} \int d \mathbf{Q} \frac{1}{4 \pi} \\
& \times\left\{\sum_{l=1}^{\infty} 2 l r_{1}^{2 l-1} \sum_{m}\left|f_{l m}\left(r_{1}, \mathbf{r}_{2}, \mathbf{Q}\right)\right|^{2}\right. \\
& \left.+\sum_{l=0}^{\infty} r_{1}^{2 l} \sum_{m} \frac{\partial\left|f_{l m}\left(r_{1}, \mathbf{r}_{2}, \mathbf{Q}\right)\right|^{2}}{\partial r_{1}}\right\},
\end{aligned}
$$

together with the result

$$
\left.\frac{\partial\left|f_{l m}\left(r_{1}, \mathbf{r}_{2}, \mathbf{Q}\right)\right|^{2}}{\partial r_{1}}\right|_{r_{1}=0}=\frac{2 \gamma}{l+1}\left|f_{l m}\left(0, \mathbf{r}_{2}, \mathbf{Q}\right)\right|^{2}
$$

obtained from Eq. (1.11), leads, in the limit $r_{1} \rightarrow 0$, to the cusp condition for the electron-pair density

$$
\left.\frac{\partial \overline{n_{2}}\left(r_{1}, \mathbf{r}_{2}\right)}{\partial r_{1}}\right|_{r_{1}=0}=-2 Z_{A} n_{2}\left(\mathbf{0}, \mathbf{r}_{2}\right)
$$

[using Eq. (1.8)]. We note that its form is the same as in the case of the electron-number density, Eq. (1.2). In fact, we can confirm the validity of Eq. (1.2) by integrating both sides of Eq. (2.12) over $d^{3} r_{2}$ and recalling the definition of $n_{1}$ in Eqs. (2.7), (2.6).

To find the cusp condition for $\varepsilon_{\mathrm{ee}}\left(\mathbf{r}_{1} ;\left[n_{2}\right]\right)$, Eq. (2.3), we calculate the derivative of its angular average, 


$$
\begin{aligned}
\left.\frac{\overline{d \varepsilon_{\mathrm{ee}}}\left(r_{1} ;\left[n_{2}\right]\right)}{d r_{1}}\right|_{r_{1}=0}= & \int \frac{d^{2} \Omega_{1}}{4 \pi} \int d^{3} r_{2} \frac{\partial}{\partial r_{1}}\left(u\left(r_{1} \mathbf{e}\left(\Omega_{1}\right), \mathbf{r}_{2}\right)\right. \\
& \left.\times n_{2}\left(r_{1} \mathbf{e}\left(\Omega_{1}\right), \mathbf{r}_{2}\right)\right)_{r_{1}=0} \\
= & \int d^{3} r_{2} \int \frac{d^{2} \Omega_{1}}{4 \pi} \\
& \times\left(n_{2}\left(\mathbf{0}, \mathbf{r}_{2}\right) \frac{\partial u\left(r_{1} \mathbf{e}\left(\Omega_{1}\right), \mathbf{r}_{2}\right)}{\partial r_{1}}\right. \\
& \left.+u\left(\mathbf{0}, \mathbf{r}_{2}\right) \frac{\partial n_{2}\left(r_{1} \mathbf{e}\left(\Omega_{1}\right), \mathbf{r}_{2}\right)}{\partial r_{1}}\right)_{r_{1}=0}
\end{aligned}
$$

But, for the Coulombic interaction,

$$
\left.\frac{\partial u\left(r_{1} \mathbf{e}\left(\Omega_{1}\right), r_{2} \mathbf{e}\left(\Omega_{2}\right)\right)}{\partial r_{1}}\right|_{r_{1}=0}=\frac{\mathbf{e}\left(\Omega_{1}\right) \cdot \mathbf{e}\left(\Omega_{2}\right)}{r_{2}^{2}} .
$$

So

$$
\begin{aligned}
\left.\frac{d \overline{\varepsilon_{\mathrm{ee}}}\left(r_{1} ;\left[n_{2}\right]\right)}{d r_{1}}\right|_{r_{1}=0}= & \int d^{3} r_{2}\left(\frac{n_{2}\left(\mathbf{0}, \mathbf{r}_{2}\right)}{r_{2}^{2}} \mathbf{e}\left(\Omega_{2}\right) \cdot \overline{\mathbf{e}}\right. \\
& \left.+\left.u\left(\mathbf{0}, \mathbf{r}_{2}\right) \frac{\partial \overline{n_{2}}\left(r_{1}, \mathbf{r}_{2}\right)}{\partial r_{1}}\right|_{r_{1}=0}\right)
\end{aligned}
$$

The first term vanishes because the angular average of the unit vector is zero and for the second term the result (2.12) should be used, so, finally,

$$
\left.\frac{\overline{d \varepsilon_{\mathrm{ee}}}\left(r_{1} ;\left[n_{2}\right]\right)}{d r_{1}}\right|_{r_{1}=0}=-2 Z_{A} \varepsilon_{\mathrm{ee}}\left(\mathbf{0} ;\left[n_{2}\right]\right) .
$$
tion

The remaining two densities in Eq. (2.1) satisfy the equa-

$$
\begin{aligned}
\lim _{r_{1} \rightarrow 0} r_{1}\left(\varepsilon_{\text {kin }}\left(\mathbf{r}_{1} ;\left[\rho_{1}\right]\right)+v\left(\mathbf{r}_{1}\right) n_{1}\left(\mathbf{r}_{1}\right)\right) & \\
& =\lim _{r_{1} \rightarrow 0} r_{1} \varepsilon_{\text {kin }}\left(\mathbf{r}_{1} ;\left[\rho_{1}\right]\right)-Z_{A} n_{1}(\mathbf{0})=0,
\end{aligned}
$$

which follows directly from the eigenequation (1.10), when the definitions (1.7), (1.8), and (2.2) are taken into account. The result (2.17) demonstrates that, at the nuclear position $\mathbf{R}_{A}=\mathbf{0}$, the singularity of the kinetic energy density cancels exactly the singularity of the electron-nucleus interaction energy density.

For DFT applications the classical electrostatic energy of electrons is of interest:

$$
E_{\mathrm{es}}\left[n_{1}\right]=\int d^{3} r_{1} \varepsilon_{\mathrm{es}}\left(\mathbf{r}_{1} ;\left[n_{1}\right]\right),
$$

where the energy density $\varepsilon_{\text {es }}$, defined in analogy with $\varepsilon_{\text {ee }}$ [Eq. (2.3)], is

$$
\varepsilon_{\mathrm{es}}\left(\mathbf{r}_{1} ;\left[n_{1}\right]\right)=\int d^{3} r_{2} u\left(\mathbf{r}_{1}, \mathbf{r}_{2}\right) \frac{1}{2} n_{1}\left(\mathbf{r}_{1}\right) n_{1}\left(\mathbf{r}_{2}\right)
$$

By applying the same steps as in Eqs. (2.13)-(2.15) with $n_{2}\left(\mathbf{r}_{1}, \mathbf{r}_{2}\right)$ replaced by $\frac{1}{2} n_{1}\left(\mathbf{r}_{1}\right) n_{1}\left(\mathbf{r}_{2}\right)$, and, next, using the condition (1.2), we arrive at the cusp condition

$$
\left.\frac{\overline{d \varepsilon_{\mathrm{es}}}\left(r_{1} ;\left[n_{1}\right]\right)}{d r_{1}}\right|_{r_{1}=0}=-2 Z_{A} \varepsilon_{\mathrm{es}}\left(\mathbf{0} ;\left[n_{1}\right]\right)
$$

in the same form as for the densities already considered.

\section{RESULTS FOR THE ADIABATIC CONNECTION TO THE NONINTERACTING EFFECTIVE SYSTEM}

Adiabatic connection, developed by Harris and Jones [7] (see also Parr and Yang [8], Sec. 8.5, and references therein), is a very convenient tool of DFT for obtaining characteristics of the noninteracting effective Kohn-Sham (KS) system by linking it with the fully interacting system via intermediate systems. For that purpose, a Hamiltonian depending on a parameter $\alpha \in[0,1]$ is introduced,

$$
\hat{H}^{\alpha}=\hat{T}+\hat{V}^{\alpha}+\alpha \hat{U}
$$

where $\hat{V}^{\alpha}=\sum_{i=1}^{N} v^{\alpha}\left(\mathbf{r}_{i}\right)$. This $\hat{H}^{\alpha}$, for $\alpha=1$, due to the requirement

$$
\hat{V}^{1}=\hat{V}, \quad v^{1}(\mathbf{r})=v(\mathbf{r})
$$

reduces to the original Hamiltonian $\hat{H}^{1}=\hat{H}$ [Eq. (1.4)], while for $\alpha=0$ it represents the noninteracting system ( $\hat{U}$ is switched off) characterized by the KS potential

$$
v^{0}(\mathbf{r})=v_{\mathrm{KS}}(\mathbf{r}) \text {. }
$$

From the eigenfunction $\Psi^{\alpha}$ of the Schrödinger equation

$$
\hat{H}^{\alpha} \Psi^{\alpha}=E^{\alpha} \Psi^{\alpha},
$$

the electron density $n_{1}^{\alpha}(\mathbf{r})$ can be calculated like $n_{1}(\mathbf{r})$ in Eqs. (2.4)-(2.7) from $\Psi$. The effective one-body potential $v^{\alpha}(\mathbf{r})$ is defined by the requirement that the electron density of the "intermediate" system remains independent of $\alpha$ :

$$
n_{1}^{\alpha}(\mathbf{r})=n_{1}^{1}(\mathbf{r})=n_{1}(\mathbf{r}) \text {. }
$$

In order to investigate the cusp conditions for the intermediate system, let us suppose that the properties of $v^{\alpha}(\mathbf{r})$ (of unknown explicit form) allow for a finite value of the limit

$$
\gamma^{\alpha}=\lim _{r_{1} \rightarrow 0} r_{1}\left(\hat{V}^{\alpha}+\alpha \hat{U}\right)=\lim _{r_{1} \rightarrow 0} r_{1} v^{\alpha}\left(\mathbf{r}_{1}\right)
$$


the analog of Eq. (1.8). Then, with $\Psi, f_{l m}, \gamma$ replaced by $\Psi^{\alpha}, f_{l m}^{\alpha}, \gamma^{\alpha}$, Eqs. (1.9)-(1.11) hold, and, therefore, all previous cusp conditions can be written for $\alpha$-dependent densities in terms of $\gamma^{\alpha}$, in particular,

$$
\left.\frac{d \overline{n_{1}^{\alpha}}(r)}{d r}\right|_{r=0}=2 \gamma^{\alpha} n_{1}^{\alpha}(\mathbf{0}),
$$

the analog of Eq. (1.2). In view of the requirement (3.5), we conclude from Eqs. (3.7) and (1.2) that

$$
\gamma^{\alpha}=\gamma=-Z_{A},
$$

what justifies a posteriori our supposition in Eq. (3.6). Therefore all cusp conditions obtained at $\alpha=1$, Eqs. (1.2), (2.12), (2.16), (2.20), hold for $n_{1}^{\alpha}, n_{2}^{\alpha}, \varepsilon_{\mathrm{ee}}^{\alpha}, \varepsilon_{\mathrm{es}}^{\alpha}$, at any $\alpha$ $\in[0,1]$.

Let us represent the intermediate-system potential as a sum of the original potential due to clamped nuclei and an additional potential that takes into account effective interactions (int) between electrons

$$
v^{\alpha}(\mathbf{r})=v(\mathbf{r})+v_{\mathrm{int}}^{\alpha}(\mathbf{r}) .
$$

Then from Eqs. (3.8), (3.6), and (1.8) it follows that the singularity of $v_{\text {int }}^{\alpha}(\mathbf{r})$ at $\mathbf{R}_{A}=\mathbf{0}$ (if it occurs at all) must be weaker than the Coulombic singularity:

$$
\lim _{r_{1} \rightarrow 0} r_{1} v_{\text {int }}^{\alpha}\left(\mathbf{r}_{1}\right)=0 .
$$

It should be noted that the $v_{\text {int }}^{0}$ part of the KS potential, Eqs. (3.3),(3.9), is traditionally split into the electrostatic (Hartree) and exchange-correlation contributions:

$$
v_{\mathrm{KS}}(\mathbf{r})=v(\mathbf{r})+v_{\mathrm{int}}^{0}(\mathbf{r})=v(\mathbf{r})+v_{\mathrm{es}}(\mathbf{r})+v_{\mathrm{xc}}(\mathbf{r}),
$$

where $v_{\text {es }}\left(\mathbf{r} ;\left[n_{1}\right]\right)=\int d^{3} r^{\prime} u\left(\mathbf{r}, \mathbf{r}^{\prime}\right) n_{1}\left(\mathbf{r}^{\prime}\right)$. Evidently, the electrostatic potential $v_{\mathrm{es}}\left(\mathbf{r} ;\left[n_{1}\right]\right)$ is finite everywhere. Therefore, Eq. (3.10) at $\alpha=0$ leads to

$$
\lim _{r_{1} \rightarrow 0} r_{1} v_{\mathrm{xc}}\left(\mathbf{r}_{1}\right)=0
$$

-an exact property of the exchange-correlation potential at the nucleus position $\mathbf{R}_{A}=\mathbf{0}$.

In the KS approach to DFT, the total molecular energy, Eq. (2.1), is rewritten as

$$
E=T_{\mathrm{s}}+V+E_{\mathrm{es}}+E_{\mathrm{x}}+E_{\mathrm{c}}
$$

(see, e.g., Parr and Yang [8]), where $T_{\mathrm{s}}=T^{0}=\int d^{3} r \varepsilon_{\text {kin }}^{0}(\mathbf{r})$ is the kinetic energy of the noninteracting $(\alpha=0)$ system, $E_{\mathrm{es}}$ is given in Eqs. (2.18) and (2.19), the exchange energy $E_{\mathrm{x}}$ is defined as

$$
E_{\mathrm{x}}=E_{\mathrm{ee}}^{0}-E_{\mathrm{es}}=\int d^{3} r\left[\varepsilon_{\mathrm{ee}}^{0}(\mathbf{r})-\varepsilon_{\mathrm{es}}(\mathbf{r})\right]=\int d^{3} r \varepsilon_{\mathrm{x}}(\mathbf{r}),
$$

and the correlation energy, given by

$$
E_{\mathrm{c}}=E_{\mathrm{ee}}^{1}-E_{\mathrm{ee}}^{0}+T^{1}-T^{0},
$$

can also be written in terms of the adiabatic-connection average (aa)

$$
E_{\mathrm{c}}=\int d^{3} r \varepsilon_{\mathrm{c}}(\mathbf{r})=\int d^{3} r\left[\varepsilon_{\mathrm{ee}}^{\mathrm{aa}}(\mathbf{r})-\varepsilon_{\mathrm{ee}}^{0}(\mathbf{r})\right],
$$

where

$$
\varepsilon_{\mathrm{ee}}^{\mathrm{aa}}(\mathbf{r})=\int_{0}^{1} d \alpha \varepsilon_{\mathrm{ee}}^{\alpha}(\mathbf{r})
$$

Here $\varepsilon_{\mathrm{ee}}^{\alpha}(\mathbf{r})=\varepsilon_{\mathrm{ee}}\left(\mathbf{r} ;\left[n_{2}^{\alpha}\right]\right)$, Eq. (2.3). Knowing that the cusp conditions hold for $\varepsilon_{\text {ee }}^{\alpha}(\mathbf{r})$ at any $\alpha$, and for $\varepsilon_{\text {es }}(\mathbf{r})$, Eqs. (2.16), (2.20), we can immediately obtain the cusp condition for the exchange energy density [see Eq. (3.14)],

$$
\left.\frac{d \overline{\varepsilon_{\mathrm{x}}}\left(r_{1}\right)}{d r_{1}}\right|_{r_{1}=0}=-2 Z_{A} \varepsilon_{\mathrm{x}}(\mathbf{0}),
$$

and for the correlation energy density [see Eqs. (3.16),(3.17)],

$$
\left.\frac{d \overline{\varepsilon_{\mathrm{c}}}\left(r_{1}\right)}{d r_{1}}\right|_{r_{1}=0}=-2 Z_{A} \varepsilon_{\mathrm{c}}(\mathbf{0}) .
$$

\section{DISCUSSION AND CONCLUSIONS}

It is worth stressing that all results for the cusp conditions are obtained from an arbitrary eigenfunction of $\hat{H}$, Eq. (1.4), or $\hat{H}^{\alpha}$, Eq. (3.1); therefore they are valid not only for the ground state (GS) of the system, but also for an arbitrary excited state. While the DFT was formulated originally for the GS, various generalizations for excited states are available now too. Thus some of our results such as Eqs. (3.12), (3.18), and (3.19) may prove to be useful for development of approximate density functionals for both the GS and excited state versions of DFT: the quality of an approximation may be checked against fulfillment of these results, or these results may be imposed as constraints during construction of approximate functionals.

The DFT approach to a single excited state proposed recently by Nagy [5] is based on the arguments that the cusp condition holds for the density $n_{1}$ corresponding to an excited state and that it remains valid also during the adiabatic connection to the effective KS system that describes this excited state. However, these arguments were criticized recently by Moiseyev [9]. Actually, he quotes reasoning from Gross [10] that Nagy's arguments cannot be true for the effective KS system, because the KS one-electron potential does not belong to the class of bare nuclear Coulomb potentials (Nagy's argument is valid only for that class, according to his opinion). Our reasoning, given in Eqs. (3.6)-(3.8), supports Nagy's arguments and shows that there is no foundation for Gross' reservation.

From the fact that the results for the cusp conditions are valid for an arbitrary eigenstate of the system, it follows also 
that they remain valid if the pure-system densities are replaced by ensemble densities. Ensemble DFT is used for describing a system at a finite temperature (see, e.g., [8]), for obtaining excited state energies [11], or for handling degeneracy of either the GS or excited states [5], and so on. An ensemble $2 \mathrm{DM}$ is defined as

$$
\rho_{2}=\sum_{\Lambda} p_{\Lambda} \rho_{\Lambda, 2}, \quad p_{\Lambda} \geqslant 0, \quad \sum_{\Lambda} p_{\Lambda}=1
$$

where $\Lambda$ denotes a set of quantum numbers of a particular eigenfunction $\Psi_{\Lambda}$, from which $\rho_{\Lambda, 2}$ is derived according to Eq. (2.4). From this ensemble 2DM other ensemble DMs are obtained according to Eqs. (2.5)-(2.7), and they satisfy the cusp conditions (1.2) and (2.12). The same is true for the ensemble $\alpha$-dependent densities. Since all considered energy densities depend on DMs linearly, the densities $\varepsilon_{\mathrm{ee}}, \varepsilon_{\mathrm{ee}}^{\alpha}, \varepsilon_{\mathrm{c}}$ for ensembles satisfy the cusp conditions (2.16),(3.19) and the density $\varepsilon_{\text {kin }}+v n_{1}$ satisfies the relation (2.17). After calculating $\varepsilon_{\mathrm{es}}$ in terms of the ensemble $n_{1}$, Eq. (2.19), the exchange energy density for an ensemble $\varepsilon_{\mathrm{x}}=\varepsilon_{\mathrm{ee}}^{0}-\varepsilon_{\mathrm{es}}$ also satisfies the cusp condition (3.18).
The cusp conditions obtained in the present paper are satisfied not only by exact solutions of many-electron problems, but also by approximate solutions that are derived from an approximate Hamiltonian, provided its potential energy term satisfies Eq. (1.8). The Hartree-Fock approximation evidently belongs to this class. Finally, since solutions for solids can be viewed as obtained by considering solutions for larger and larger clusters, all derived cusp conditions (being local properites) remain valid for condensed matter.

In concluding, our paper demonstrates that nuclear cusp conditions are satisfied by various terms of the energy density that occur in quantum chemistry in general and in a multitude of DFT approaches.

\section{ACKNOWLEDGMENTS}

The Flemish Science Foundation (FWO) is acknowledged by I.A.H. for support under Grant No. G.0347.97 and also by P.S. for fiancial support. The University of Antwerp (RUCA) is thanked by I.A.H. for its support. This work was also supported by the Concerted Action Program of the University of Antwerp.
[1] T. Kato, Commun. Pure Appl. Math. 10, 151 (1957).

[2] W. A. Bingel, Z. Naturforsch. A 18, 1249 (1963).

[3] R. T. Pack and W. B. Brown, J. Chem. Phys. 45, 556 (1966).

[4] E. Steiner, J. Chem. Phys. 39, 2365 (1963).

[5] Á. Nagy, Int. J. Quantum Chem. 70, 681 (1998).

[6] E. R. Davidson, Reduced Density Matrices in Quantum Chemistry (Academic Press, New York, 1976).

[7] J. Harris and R. O. Jones, J. Phys. F: Met. Phys. 4, 1170
(1974).

[8] R. G. Parr and W. Yang, Density-Functional Theory of Atoms and Molecules (Oxford University Press, New York, 1989).

[9] N. Moiseyev, Chem. Phys. Lett. 321, 469 (2000).

[10] E. K. U. Gross (unpublished), quoted in [9].

[11] E. K. U. Gross, L. N. Oliveira, and W. Kohn, Phys. Rev. A 37, 2805 (1988); 37, 2809 (1988). 